\title{
Bemerkung zu Dr. M. Sugárs Aufsatz: Labyrinth und Nystagmus.
}

\author{
Von Privatdozent Dr. Huge Frey, Wien. \\ In Bd. 81 , Heft 1 dieses Archivs.
}

Herr Dr. Sugár nimmt eine auf dem KongreB in Budapest im August vergangenen Jahres stattgefundene Diskussion zum Anlaß, um die Verdienste Högy es um die Physiologie des Vestibularapparates in einem Aufsatz klarzulegen - zweifellos ein dankenswertes Unternehmen, wenn auch die Arbeiten $H o ̈ g y e s$ wohl allen, die sich für die hiehergehörigen Fragen interessieren, bekannt sind.

Er greift aber bei dieser Gelegenbeit auf die erwähnte Diskussion selbst zurück und behauptet, ich hätte in derselben "auf das Bestimmteste" erklärt, "von $\mathrm{H}$ ög yes und seinen diesbezüglichen Arbeiten nichts zu wissen".

Leider liegen die Verhandlungen des Kongresses noch nicht vor, es wăre sonst sehr leicht, den Nachweis zu führen, daß ich eine derartige Außerung nicht gemacht habe. Das konnte mir auch um so weniger einfallen, als mir die verschiedenen Arbeiten Högyes wohl bekannt sind. Aber es scheint, dab ich entweder von Herra Dr. Sugár ganz gründlich miBverstanden warde, oder daB sich in seiner Erinnerung das Bild der damaligen Diskussion wesentlichst verschoben hat.

Es handelte sich um die Funktionsprüfung des Vestibularapparates in bezug auf praktische klinische Fragen und die diagnostische Methodik. Hier wollte Dr. Sugár auch das Verdienst für diese, rein klinische Angelegenheit Hŏgyes zugeteilt wissen. Demgegenüber bemerkte ich nun, daß gewißj niemand behaupten würde, die Physiologie des Vestibulapparates und dessen Beziehungen zu den Augenmuskeln im besonderen sei eine neue $\mathrm{Er}$ rungenschaft der letzten Zeit. Die Untersuchungstechnik des Vestibularapparates aber, deren diagnostische Verwertung und die damit zusammenhängende Beeintlussung der Therayie seien neue Dinge, von denen Högyes niemals gesprochen habe.

Diese Behauptung halte ich selbstverständlich aufrecht. Sie wird auch durch keine Zeile des Sugárschen Aufsatzes entkräftet. 